\title{
Analysis of intensive care populations to select possible candidates for high dependency care
}

\author{
J V Pappachan, B W Millar, D J Barrett, G B Smith
}

\begin{abstract}
Objectives-To identify the proportion, and range across intensive care units, of intensive care patients who might potentially be managed on a high dependency unit (HDU) using three different classification systems.

Methods-8095 adult patients admitted to 15 intensive care units in the south of England between 1 April 1993 and 31 December 1994 were studied. Patients were identified as potential HDU admissions if their APACHE III derived risk of hospital mortality was $\leqslant 10 \%$, if they were categorised as a low risk monitor (LRM) patient using the Wagner risk stratification method, or if they did not require advanced respiratory support (ARS).

Results-4146 patients $(51.2 \%)$ had an APACHE III derived risk of hospital death of $\leqslant 10 \%, 1687(20.8 \%)$ were classified as LRM, and $3860(47.7 \%)$ did not receive ARS. The values for each intensive care unit ranged from $32.8-63.3 \%$ (APACHE III group), 7.2-29.9\% (LRM group), and $\mathbf{1 4 . 4 - 6 8 . 2 \%}$ (ARS group). No matter which of the three methods was used, there were significant differences between the 15 units $(p<0.0001)$ with regard to the number of potential HDU patients identified within the scored population.

Conclusions-The percentage of intensive care patients who might be more appropriately managed in a HDU varies considerably between hospitals, and depends upon both local circumstances and the method used to define a high dependency patient. However, whichever method is used, it appears that significant numbers of patients of low dependency status currently fill intensive care beds in the units studied. If these analyses are correct, the perceived national shortage in intensive care beds might be improved by the development of HDUs.

(F Accid Emerg Med 1999;16:13-17)
\end{abstract}

Keywords: high dependency care; intensive care; triage; patient selection
In the USA and the UK critically ill patients appear to have a better outcome if admitted to intensive care units directly from the accident and emergency (A\&E) department, ${ }^{12}$ in comparison with those from general wards. In the UK in particular, some critically ill patients will be admitted to general wards from the $A \& E$ department because of the lack of high dependency or intensive care beds.

Opinions vary concerning the use and provision of high dependency or intensive care beds. ${ }^{3-12}$ At present only approximately $15 \%$ of UK hospitals possess a high dependency unit (HDU), ${ }^{13}{ }^{14}$ yet studies from individual hospitals have suggested that up to $40 \%$ of patients currently admitted to an intensive care unit might be more appropriately managed in a HDU if one was available. ${ }^{39101215}$ Although these estimates generally rely upon subjective assessments of the need for HDU care, several objective methods have been described..$^{311} 1617$

Kilpatrick et al have suggested that patients may be safely nursed on a HDU if their predicted hospital mortality, derived using the acute physiology and chronic health evaluation II (APACHE II) scoring system, ${ }^{18}$ is $\leqslant 10 \%{ }^{3}$ This mortality prediction assumes that the patient is managed on a intensive care unit and may not be valid if the patient is nursed on a HDU instead. ${ }^{19}$

Wagner et al have described an alternative method which uses a risk stratification system. ${ }^{16}$ Intensive care unit admissions are categorised into active treatment, high risk monitor (HRM), and low risk monitor (LRM) groups; the distinction between HRM and LRM is made using the predicted risk of receiving active intensive care unit type treatment $(>10 \%$ for the HRM group and $<10 \%$ for the LRM group). Wagner et al suggest that LRM patients, who by definition have low intervention and low mortality rates, could be appropriately placed in a HDU. Some of the active treatment modalities, identified as requiring intensive care unit admission in the USA, can, in our opinion, be managed in a less dependent area in the UK and, therefore, Wagner's technique may not be transferable. In the UK, patients who do not require advanced respiratory support (ARS) - for example nasotracheal or orotracheal intubation, mechanical ventilatory support (excluding mask continuous positive airways pressure (CPAP) and 
Table 1 The list of 31 unique intensive care type interventions referred to by Wagner. ${ }^{16}$ Numbers 1 to 4 represent ARS as defined by the authors

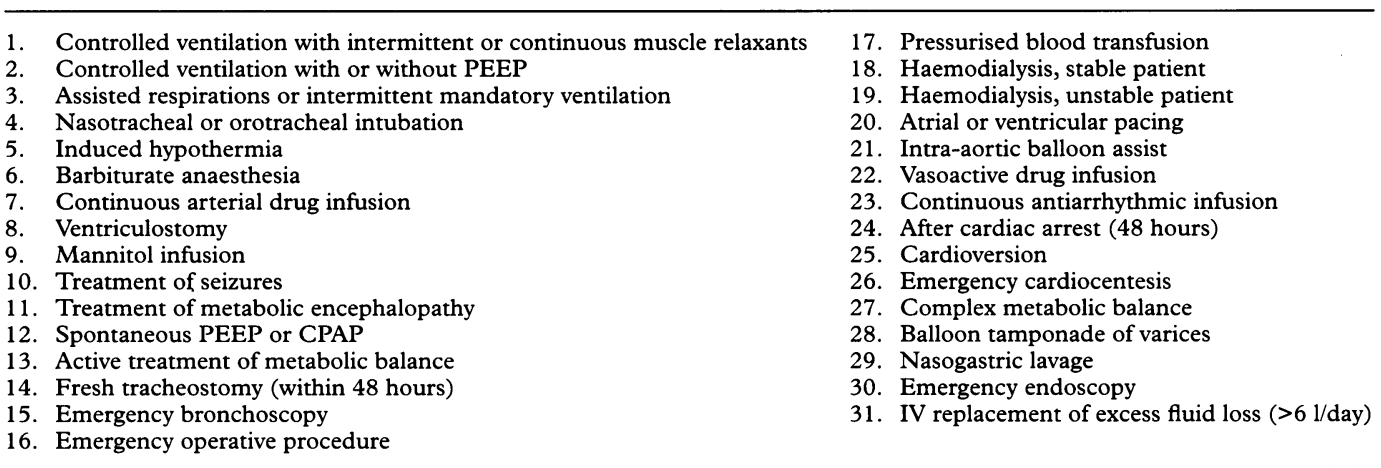

non-invasive, for example mask, ventilation)are often nursed on HDUs.

We postulated that the number of intensive care unit patients who might be classified as

Table 2 Demographic data for the scored patients in the South West Thames database in the period studied and the corresponding values for the North American APACHE III database

\begin{tabular}{|c|c|c|}
\hline Patient characteristics & $\begin{array}{l}\text { South West } \\
\text { Thames database }\end{array}$ & $\begin{array}{l}\text { American } \\
\text { APACHE III } \\
\text { database }\end{array}$ \\
\hline No of patients & 8095 & 17440 \\
\hline \multicolumn{3}{|l|}{ Sex $(\%)$} \\
\hline Male & 58.7 & 44.8 \\
\hline Female & 41.3 & 55.2 \\
\hline Age in years (mean and range) & $60.3(16-101)$ & $59(16-113)$ \\
\hline$<45(\%)$ & 20.5 & 23.2 \\
\hline $45-65(\%)$ & 29.7 & 28.8 \\
\hline $66-85(\%)$ & 46.8 & 42.7 \\
\hline$>85$ & 2.9 & 5.3 \\
\hline Severe comorbidities before admission (\%) & 7.9 & 13.0 \\
\hline \multicolumn{3}{|l|}{ Operative status (\%) } \\
\hline Non-operative & 55.3 & 57.7 \\
\hline \multicolumn{3}{|l|}{ Operative } \\
\hline Elective & 27.0 & 33.3 \\
\hline Emergency & 17.7 & 9.0 \\
\hline \multicolumn{3}{|l|}{ Severity of illness (average first day) } \\
\hline APACHE III score & 56.2 & 50 \\
\hline$\leqslant 10 \%$ predicted risk of death $(\%)$ & 51.0 & 63 \\
\hline$>90 \%$ predicted risk of death $(\%)$ & 2.3 & 2.3 \\
\hline \multicolumn{3}{|l|}{ Outcome (\%) } \\
\hline Average ICU mortality & 17.6 & 9.2 \\
\hline \multicolumn{3}{|l|}{ Length of stay (days) } \\
\hline Average ICU length of stay & 4.8 & 4.9 \\
\hline \multicolumn{3}{|l|}{ Treatment level (\%) } \\
\hline Active treatment & 71.0 & 54.0 \\
\hline HRM & 8.2 & 11.0 \\
\hline LRM & 20.8 & 35.0 \\
\hline Patients not receiving advanced respiratory support ( $\%$ ) & 50.0 & * \\
\hline
\end{tabular}

${ }^{\star}$ No data available. ICU $=$ intensive care unit.

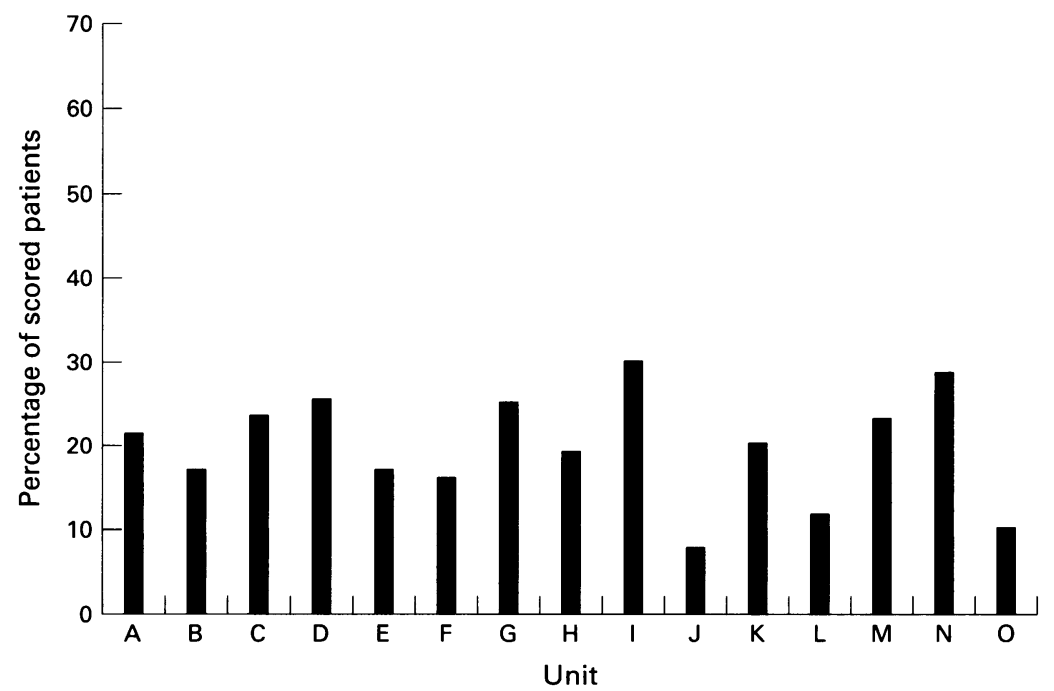

Figure 1 Percentage of scored patients with $a \leqslant 10 \%$ risk of hospital mortality as assessed by the APACHE III predictive algorithm. potential HDU admissions would vary between units and might depend upon the classification method employed. The aim of this retrospective observational study was to estimate the proportion of intensive care patients from 15 units that might have been cared for on a HDU by using the three methods outlined above.

\section{Methods}

All admissions to 15 intensive care units (labelled A to O) in the Western Division of the South Thames Regional Health Authority and the Portsmouth Health District were studied between 1 April 1993 and 31 December 1994. Four of these units (J, K, N, and O) were pure general intensive care units while the remaining 11 served as mixed intensive and coronary care units with patients from both categories being nursed in the same floor area. In 10 of these, coronary care patients were managed by general physicians, whereas in the remaining unit (I) the intensive care unit consultants took responsibility for the management of all patients on the unit. The coronary care unit patients admitted to unit I were included in the APACHE III analysis.

An identical system of data collection (Ward Watcher (1) software) and central analysis (Critical Audit Ltd) was used in all intensive care units. An assessment of risk of hospital death was made for each patient using the APACHE III scoring system ${ }^{20}$ unless they (a) were aged under 16 years; (b) had a diagnosis of primary burns injury; (c) died within four hours of intensive care unit admission; or (d) had been admitted to intensive care units $\mathrm{A}-\mathrm{H}$ and $\mathrm{J}-\mathrm{O}$ because either a separate coronary care or theatre recovery unit did not exist or was not available in the hospital concerned.

For each patient scored using the APACHE III system, demographic data including age, gender, diagnosis, reason for admission (that is, medical, elective surgical, or emergency surgical), pre-existing co-morbidity, intensive care unit length of stay, hospital length of stay, and actual hospital outcome were recorded. Data were further analysed by Critical Audit Ltd, using algorithms supplied by Apache Medical Systems Inc, to subdivide admissions into active treatment, HRM, and LRM groups. Table 1 lists active treatment tasks as defined by Wagner et al. ${ }^{16}$ 


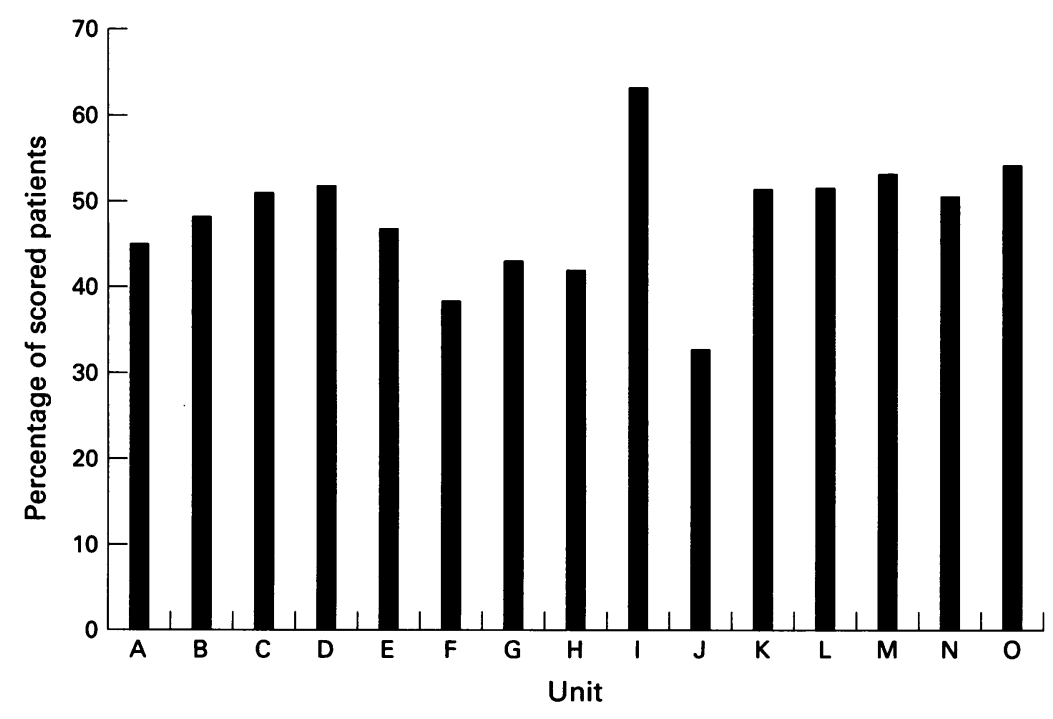

Figure 2 Percentage of scored patients in the LRM group.

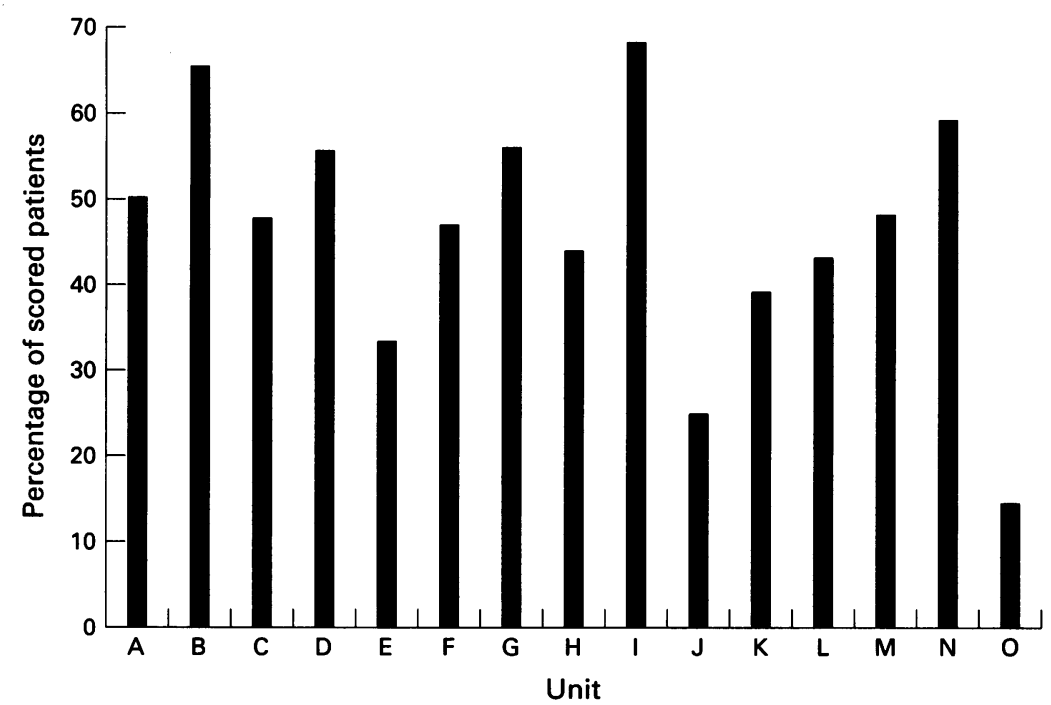

Figure 3 Percentage of scored patients not receiving ARS.

We then identified those scored patients who required ARS. This was defined as the need for mechanical ventilatory support (excluding mask CPAP and non-invasive, for example mask, ventilation), nasotracheal or orotracheal intubation.

The number of intensive care units admissions in each centre which might have been cared for in an HDU environment were then quantified using three different criteria: (a) those with a $\leqslant 10 \%$ risk of mortality using the APACHE III system (Apache III group); (b) those with a $<10 \%$ risk of requiring intensive care unit type interventions as described by Wagner et al $^{16}$ (LRM group); and (c) those not receiving ARS.

\section{Results}

A total of 15717 patients were admitted to the 15 intensive care units studied. Altogether 7622 patients were excluded from APACHE III analysis; of these $89.7 \%$ (6837) had been admitted to the unit because either a separate coronary care or theatre recovery unit did not exist or was not available in the hospital concerned. The percentage of patients excluded varied between $10.3 \%$ (unit $\mathrm{K}$ ) and $83.6 \%$ (unit $\mathrm{A}$ ).

Eight thousand and ninety five patients $(51.5 \%)$ had a prediction of hospital mortality derived using the APACHE III system and this group can be regarded as representing the "true" intensive care population. The means of the raw and derived data from the group are shown in table 2 .

Overall, the percentage of the 8095 scored intensive care unit patients who might have been suitable for HDU care was $51.2 \%$ (APACHE III group), $20.8 \%$ (LRM group), and $50.0 \%$ (ARS group). The values for each intensive care unit are shown in figs 1-3 and ranged from 32.8-63.3\% (APACHE III group), $7.2-29.9 \%$ (LRM group), and 14.4$68.2 \%$ (ARS group). The rank order of units varied depending upon the method used to identify potential HDU patients. Unit J admitted the lowest number of potential HDU patients when admissions were ranked on the basis of APACHE III risk of mortality and LRM. In contrast, unit I admitted the highest number of potential HDU patients when admissions were ranked using any of the three methods.

Individual data from each of the 15 intensive care units are shown in table 3.

There were statistically significant differences between the 15 intensive care units with respect to the percentage of scored patients admitted for medical reasons (mean $55.3 \%$, range $37.5 \%-78.7 \% ; \mathrm{p}<0.0001$ ) and after elective (mean $27 \%$, range $11.3 \%-40.6 \%$; $\mathrm{p}<0.0001$ ) and emergency operations (mean $17.7 \%$, range $10 \%-34.4 \% ; p<0.0001)$. For the purpose of this analysis we used the definitions of medical and surgical admissions in the APACHE III scoring system. ${ }^{20}$ We also showed

Table 3 Details of admissions to the 15 intensive care units studied describing the numbers admitted and scored, the number with a hospital mortality as predicted by APACHE III of $\leqslant 10 \%$, those in the LRM group, and those not receiving ARS

\begin{tabular}{|c|c|c|c|c|c|c|c|c|c|c|c|c|c|c|c|}
\hline & $A$ & $B$ & $C$ & $D$ & $E$ & $F$ & $G$ & $H$ & $I$ & $\mathcal{F}$ & $K$ & $L$ & $M$ & $N$ & $O$ \\
\hline Admissions & 1217 & 658 & 667 & 1454 & 572 & 1628 & 234 & 884 & 1488 & 445 & 435 & 1399 & 1981 & 1461 & 1194 \\
\hline Excluded & 1018 & 242 & 188 & & 25 & 033 & 106 & 68 & & & 4 & 85 & & 174 & 187 \\
\hline Scored & 199 & 416 & 479 & 497 & 319 & 595 & 128 & 201 & 1265 & 387 & 390 & 509 & 416 & 1287 & 1007 \\
\hline No in LRM group & 43 & 71 & 112 & 126 & 53 & 94 & 32 & 38 & 378 & 28 & 77 & 58 & 96 & 371 & 100 \\
\hline$\%$ & 21.5 & 17.0 & 23.4 & 25.3 & 16.6 & 15.8 & 25.0 & 18.9 & 29.9 & 7.2 & 19.7 & 11.4 & 23.0 & 28.8 & 9.9 \\
\hline $\begin{array}{l}\text { No with } \leqslant 10 \% \text { risk } \\
\text { of hospital } \\
\text { mortality }\end{array}$ & 90 & 201 & 244 & 259 & 149 & 229 & 55 & 84 & 801 & 127 & 202 & 264 & 222 & 651 & 547 \\
\hline$\%$ & 45.2 & 48.3 & 50.9 & 52.1 & 46.7 & 38.5 & 43.0 & 42.0 & & 32.8 & 51 & 51 & 53.4 & 50 & 54.3 \\
\hline $\begin{array}{l}\text { No not receiving } \\
\text { ARS }\end{array}$ & 100 & 273 & 229 & 277 & 106 & 279 & 72 & 88 & 863 & 95 & 153 & 219 & 200 & 761 & 145 \\
\hline$\%$ & 50.3 & 65.6 & 47.8 & 55.7 & 33.2 & 46.9 & 56.2 & 43.8 & 68.2 & 24.5 & 39.2 & 43 & 48.1 & 59.1 & 14.4 \\
\hline
\end{tabular}


a significant difference between the numbers of patients excluded from APACHE III analysis in each unit $(\mathrm{p}<0.0001)$. In addition, no matter which of the three methods was used, there were significant differences between the 15 units $(p<0.0001)$ with regard to the number of potential HDU patients identified within the scored population.

\section{Discussion}

The inappropriate placement of high dependency patients in intensive care beds may lead to an increase in costs, greater numbers of patient transfers between intensive care units, delays in transferring critically ill patients from A\&E units, and the cancellation of major routine surgery.$^{6-8}$ However, most methods of identifying HDU patients from within the intensive care unit population are subjective which may make categorisation invalid. A small number of publications suggest specific objective criteria, ${ }^{311} 1617$ but these may also produce differing estimates.

Henning et al used an acute physiology score $\leqslant 10,{ }^{17}$ but this technique fails to recognise the important contribution of diagnosis to the risk of mortality. Furthermore it lacks discrimination because there is considerable overlap in the degree of physiological derangement between ward and intensive care patients. ${ }^{21}{ }^{22} \mathrm{Kil}-$ patrick et al used an APACHE II derived mortality prediction of $\leqslant 10 \%$ as the arbiter for selection of HDU patients. ${ }^{3}$ This technique may overestimate the true number of patients who could safely be transferred from an intensive care unit, ${ }^{5}$ because the APACHE II predictive equation assumes that subsequent care will be in an intensive care unit and not an HDU. ${ }^{19}$ Wagner et $a l^{16}$ described a risk stratification method which, if employed in the UK, might underestimate the number of HDU patients within an intensive care unit, because some of the interventions considered to require intensive care unit admission in the USA could be managed safely in a less dependent area. Moreover, some are esoteric and are infrequent treatment modalities on a general adult intensive care unit in the UK.

Other publications have used the number and chronicity of failing organs, or the requirement for organ support, as indicators of the need for HDU or intensive care unit admission. ${ }^{11}{ }^{23-25}$ Common to all is the view that patients requiring ARS (mechanical ventilation with or without intubation) should be admitted to an intensive care unit. For this reason, we proposed that patients who do not require ARS might be suitable for high dependency, rather than intensive, care. Nevertheless, we recognise fully that a small number of patients, requiring only basic respiratory support, might need intensive care unit admission for other reasons.

All three methods tested in our study are flawed as arbiters of the requirement for intensive care over time. The APACHE III method and the LRM technique of Wagner et al estimate the need for intensive care based on the severity of illness within the first 24 hours of intensive care unit admission. The third method used in our study stratified patients according to their need for ARS at any point during intensive care unit admission and may therefore have overestimated the true requirement for intensive care facilities. These flaws may be corrected when augmented care period data become available. ${ }^{26}$ This method, recently devised by the NHS Executive, is likely to provide information regarding the number of intensive care unit bed days occupied by high dependency patients.

We have shown that the percentage of intensive care unit patients who might be better managed in a HDU varies considerably between hospitals and with local circumstances. However, this figure also depends heavily upon the method used to define a high dependency patient. Overall, 4146 (51.2\%) intensive care admissions had a risk of hospital mortality of $\leqslant 10 \%, 1687(20.8 \%)$ fell into the LRM category, and $3860(47.7 \%)$ did not require ARS. Using these figures, the number of scored intensive care unit patients who would be classed as high dependency patients ranges from 1687 to 4146 . Alarmingly, this would classify between $59 \%$ and $75 \%$ of our original population of 15717 patients (7622 of which were not scored) as potential HDU admissions. Equally concerning is the discovery that six of the 15 intensive care units studied (that is units $A, B, E, G, H$, and $M$ ) appear to admit fewer than 200 "true" intensive care patients per annum and are clearly already working predominantly as HDUs, if one adopts the classification proposed by the Intensive Care Society. ${ }^{24}$ At the other end of the spectrum, unit $O$ scored 1007 of 1194 patients $(84.3 \%)$ admitted during the study period, and, of these, $862(85.6 \%)$ required ARS.

It is estimated that, in the UK, approximately 10000 transfers of critically ill patients occur annually, many simply because of a lack of intensive care facilities in the base hospital. ${ }^{27}$ In addition, many patients requiring postoperative intensive care have surgery postponed because intensive care beds are unavailable. ${ }^{28}$ Possibly more worryingly, the proportion of intensive care patients who are admitted directly from $A \& E$ is significantly smaller than in the USA $(23.5 \% v 35.8 \%)^{2}$ and this may be a contributing factor to the apparent excess hospital mortality observed when intensive care practice in southern England is compared with that in the USA. ${ }^{2}$ This admission of ill patients from an A\&E department to a general ward may be the understandable result of an absence or relative deficiency of high dependancy or intensive care beds in a hospital, but it is recognised that such patients may receive substandard care which may contribute to increased morbidity and mortality. ${ }^{29}$

The finding that approximately $50 \%$ of scored patients in 15 intensive care units in the south of England might require only high dependency care could suggest that the UK possesses adequate numbers of intensive care beds, but that their correct use is often hampered by a lack of high dependency facilities. The solution almost certainly involves a combination of actions. Intensive care beds, 
which are used predominantly for high dependency care, should be reclassified and restaffed as high dependency beds. In addition, intensive care beds should be redistributed to those hospitals that already cancel a significant number of major acute surgical procedures and/or undertake many interhospital transfers because of a lack of local intensive care resources, after ensuring that these are not the same hospitals that fill intensive care beds with HDU patients. There may also need to be an expansion of HDUs to cater for the significant number of patients on the general wards who might also benefit from this level of care ${ }^{4}$ and who have not been considered by our study.

We would like to thank the Directors and staff of the intensive care units of the South West Thames Intensive Care audit group for their permission to use data from their units.

1 Escarce JJ, Kelley MA. Admission source to the medical intensive care unit predicts hospital death independent of APACHE II score. $\Im A M A 1990 ; 14: 2389-94$.

2 Pappachan JV, Millar B, Bennet ED, et al. Comparison of outcome from intensive care admission after adjustment for case mix by the APACHE III prognostic system-a study of 17 intensive care units in the South of England. Chest 1998 (in press)

3 Kilpatrick A, Ridley S, Plenderleith L. A changing role for intensive therapy; is there a case for high dependency care? intensive therapy; is there a case
Anaesthesia 1994;49:666-70.

4 Leeson-Payne CG, Aitkenhead AR. A prospective study to assess the demand for a high dependency unit. Anaesthesia 1995;50:383-7.

5 Pappachan VJ, Smith GB, Taylor BL, et al. HDU care and estimated risks of hospital mortality. Anaesthesia 1995;50 657-8.

6 Ryan DW. High dependency units may be the answer. $B M F$ 1995;310:1010-1.

7 Smith GB, Taylor BL, McQuillan PJ. Rationing intensive care. Intensive care provision varies widely in Britain. $B M{ }^{\prime}$ $1995 ; 310: 1412-3$.

8 Peacock JE, Edbrooke DL. Rationing intensive care. Data from one high dependency unit supports their effectiveness. $B M F$ 1995;310:1413.

9 Crosby DL, Rees GAD. Provision of postoperative care in UK hospitals. Ann R Coll Surg Engl 1994;76:14-18.

10 Ryan W, Bayly PJM, Weldon OGW, et al. A prospective twomonth audit of the lack of provision of a high-dependency month audit of the lack of provision of a high-dependency 265-75.
11 Department of Health. Guidelines on admission to and discharge from intensive care and high dependency units. London: Department of Health, 1996.

12 Bodenham AR, Knappett P, Cohen A, et al. Facilities and usage of general intensive care in Yorkshire. A need for high-dependency units. Clinical Intensive Care 1995;6:260 5.

13 High dependency units in the UK: variable size, variable character, few in number. Postgrad Med f 1995;71:217-21.

14 Royal College of Anaesthetists. Royal College of Anaesthetist national ITU audit 1992/1993. London: RCA, 1994.

15 Metcalfe A, McPherson K. Study of provision of intensive care in England, 1993. Revised report for the Department of Health. London: Department of Health, January 1995.

16 Wagner DP, Knaus WA, Draper EA. Identification of low-risk monitor admissions to medical-surgical ICUs. Chest 1987;92:423-8.

17 Henning RJ, McClish D, Daly B, et al. Clinical characteristics and resource utilization of ICU patients: implications for organisation of intensive care. Crit Care Med 1987;15: 264-9.

18 Knaus WA, Draper EA, Wagner DP, et al. APACHE II; a severity of disease classification system. Crit Care Med 1985;13:818-29.

19 Lemeshow S, Teres D, Klar J, et al. Mortality probability models (MPM II) based on an international cohort of intensive care unit patients. $\mathcal{F} A M A$ 1993;270:2478-86.

20 Knaus WA, Wagner DP, Draper EA, et al. The APACHE II prognostic system. Risk prediction of hospital mortality for critically ill hospitalised adults. Chest 1991;100:1619-36.

21 Franklin C, Rackow EC, Mamdani B, et al. Triage considerations in medical intensive care. Arch Intern Med 1990;150:1455-9.

22 McClisk DK, Russo A, Franklin C, et al. Profile of medical ICU vs ward patients in an acute care hospital. Crit Care Med 1985;13:381-6.

23 Association of Anaesthetists of Great Britain and Ireland The high dependency unit-acute care in the future. London: Association of Anaesthetists of Great Britain and Ireland, 1991.

24 Intensive Care Society. The intensive care service in the UK. London: Intensive Care Society, 1990.

25 Bion JF. Audit and quality assurance in critical care. In Dobb GJ, Bion JF, Burchardi $\mathrm{H}$, et al, eds. Current topics in intensive care 1. London: WB Saunders, 1994.

26 Committee for Regulating Information Requirements. Data set change notice 29/96/P24. Augmented care period data set. Birmingham: NHS Executive, 1996.

27 Mackenzie PA, Smith EA, Wallace PGM. Transfer of adult between intensive care units in the United Kingdom: postal survey. $B M \mathcal{F}$ 1997;314:1455-6.

28 Smith GB, Taylor BL, McQuillan PJ, et al. Rationing intensive care [letter]. BMF 1995;310:1412-3.

29 McQuillan P, Pilkington S, Allen A, et al. A confidential enquiry into the quality of care prior to intensive care admission. $B M \mathcal{F}$ 1998;316:1853-58. 